\title{
53. Neocortical Response to Odors of Sex Steroid Hormones in the Dog
}

\author{
By Norihiko Onoda, Takeshi ARIKI, Kazuyuki Imamura, \\ and Masae IINo \\ Department of Physiology, School of Medicine, \\ Gunma University, Maebashi 371 \\ (Communicated by Yasuji KatsUKi, M. J. A., Sept. 13, 1982)
}

Recent physiological studies have demonstrated that oligosynaptic neuronal systems arise from the olfactory bulb and terminate in the orbitofrontal cortex in the primate ${ }^{12), 13)}$ and other mammals..$^{6)-8)}$ In the latter, it has been found that neocortical neurons responded selectively to odors of biologically derived products which were thought to contain some pheromonal substances. Although sex steroid hormones were presumed to be mammalian pheromones, neural responses to odors of sex steroids were reported in only the olfactory bulb of the miniature pig.5) The main objective of this paper is to show single unit response of the orbital gyrus (ORG) to steroids in the dog.

Seven adult dogs of both sexes ( $4-7 \mathrm{~kg}$ ) were used. A specially designed head attachment was fixed to the animal's skull.8) A week after the initial surgery, double cannulation for artificial ventilation and artificial air intake was carried out. Fine teflon wires were tightly inserted into the nasopalatine ducts on both sides to rule out the vomeronasal input. The animal's head was immobilized on a sterotaxic frame by fixing the head attachment to an overhead bar. The dorsal surface of the frontal cortex was exposed. These operations were performed under Na-pentobarbital anesthesia $(30 \mathrm{mg} / \mathrm{kg}$, i.v. $)$.

About six hrs after the application of Na-pentobarbital, the animal was paralyzed with Br-pancuronium ( $1 \mathrm{mg} / \mathrm{kg} / \mathrm{hr}$, i.v.) and artificially ventilated. End-tidal $\mathrm{CO}_{2}$ was maintained between 3.5 and $4.5 \%$. Local anesthetic was periodically applied to the wounded tissues. Extracellular single unit responses of neocortical neurons were recorded by glass micropipettes filled with a blue dye. ${ }^{2)}$ All unit activity was stored on magnetic tape for later statistical processings. Air intake into the nasal cavity was induced by applying negative pressure pulses (300 msec duration, $1 \mathrm{~Hz})$ and its time course was monitored with a pressure transducer. ${ }^{8)}$

The animal's own fresh urine and feces, conspecific fresh urine and feces, and four steroids (Sigma, St Louis, Missouri) - progester- 


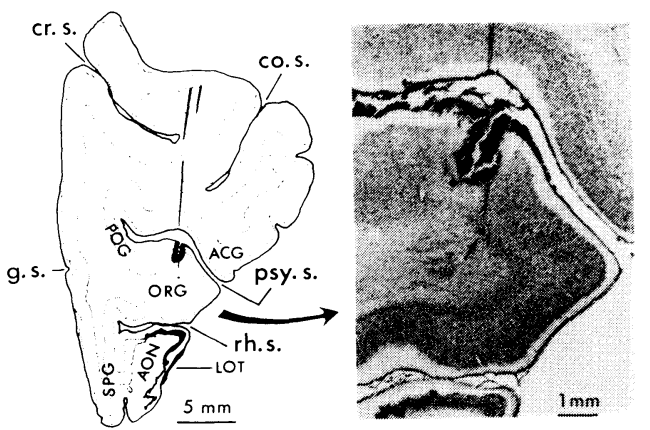

Fig. 1. A typical example of unit location. On the left, a whole view of the frontal section. On the right, the magnified ORG. The small arrow indicates a spot marked by dye deposition. Extensive haemorrhage is seen over the spot. Anatomical abbreviations: ACG, anterior composit gyrus; AON, anterior olfactory nucleus; co.s., coronal sulcus; cr.s., cruciate sulcus; g.s., genual sulcus; LOT, lateral olfactory tract; ORG, orbital gyrus; POG, paraorbital gyrus; psy.s., presylvian sulcus; rh.s., rhinal sulcus; SPG, subprorean gyrus.

A

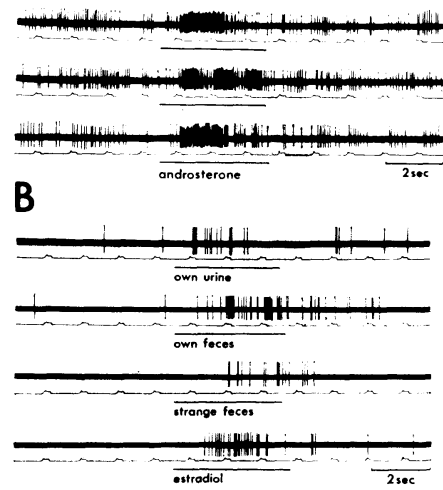

Fig. 2. Single unit responses to odors. The line below each record indicates the period of odor application. The middle trace shows output of a pressure transducer. Upward is the negative direction associated with air intake. A: Repeated facilitatory responses of an $\mathrm{ORG}$ neuron to a steroid odor. B: Facilitatory responses of another neuron to different odors. Each steroid was mixed with an odorless mineral oil (Plough Inc. Memphis, Tenn.), sonicated, evaporated, and centrifuged. $200 \mu \mathrm{l}$ of a saturated steroid solution and a few drops of animal products were stored in a separate $30 \mathrm{ml}$ syringe. Syringe plungers were pushed by a motor controlled by a pulse generator. Each odor stimulation was applied to the animal's nostrils through teflon tubing for 3-5 sec.

one, estradiol, testosterone, and androsterone-were used as odor stimuli. Each odor stimulation was randomly repeated at least three times at 1 to $3 \mathrm{~min}$ intervals. Deodorized air stream $(6 \mathrm{l} / \mathrm{min})$, to which a lack of response was verified, was always flowed to the animal's nostrils.

For identification of neurons which responded to odors, a DC current was passed through each recording point, and blue spots produced by a dye from histological sections were explored later.

We have examined the neural response to odor stimuli in more than several hundreds units. Most of the neurons did not respond to any of the odors. Twenty-four units, however, showed significant changes (Mann-Whitney's U-test, $\mathrm{P}<0.01$ or Student's t-test, $\mathrm{P}<0.01$ ) 
in their discharge rate during the application of at least one of the specific odors. ${ }^{\top 1}$ A typical example of their recording points is shown in Fig. 1. The left section shows that an electrode track penetrating the anterior composit gyrus reaches the dorsal bank of the ORG, and that a spot of blue dye deposition is seen beneath the end of the track. The right magnified photo shows that the spot indicated by the small arrow is located in the fifth layer of the ORG. From the histological check, it was confirmed that the location of units occupied a restricted region of the $\mathrm{ORG}^{\top}$ ) and that according to Kreiner's classification, it belonged to ORB II a and b. ${ }^{4}$

Out of 24,14 ORG units $(58.3 \%)$ responded to at least one of the steroids. A typical example of unit activity is shown in Fig. 2A. It can be seen that each discharge rate increased with repeated application of the androsterone odor. This unit was not affected by other odors. Responses of another neocortical neuron are shown in Fig. 2B. This unit showed a facilitatory response to odors of the feces and the urine, and the estradiol odor, but no response to others.

Neocortical responses obtained from 14 units to steroids are shown in Fig. 3. It appears that all of the units recorded show

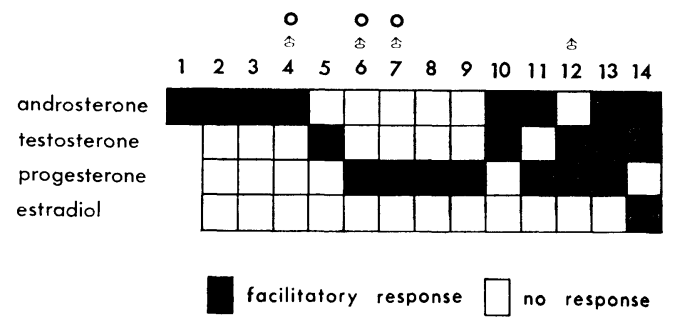

Fig. 3. A panel histogram obtained from 14 ORG units. The number at the top indicates the unit numbers arranged in the order of the number of odors responded to. Open circles over the unit numbers show the unit which responded to one steroidal odor alone. $\uparrow$ denotes male dog.

facilitatory responses to odors of steroids. It can be seen that the number of units which responded to one steroid was the largest. In fact, out of 14,9 units $(64.3 \%)$ and 3 units $(21.4 \%)$ responded to one steroid and to two, respectively. No particular relationship between neocortical responses to steroids and the sex of the animal could be found in this experiment. Out of 14, three units $(21.4 \%)$, indicated by open circles over the unit number in Fig. 3, responded exclusively to one steroid odor, while the other units $(78.6 \%)$ responded to at least one of the odors of animal products such as urine and feces. 
As reported in previous papers, a particular neuron of the orbitofrontal cortex showed the highly selective response to odors in the rabbit, ${ }^{6)}$ dog, ${ }^{7)}$ and monkey. ${ }^{12), 13)}$ In addition, recent behavioral studies demonstrated that animals with lesions of the orbitofrontal cortex had their odor preferences and sexual behavior impaired.1,11) And also we found that a particular ORG neuron responded exclusively to steroid odors. Thus, in the dog, steroids would work as the ectohomones which communicate olfactory messages such as sex differences and the sexual state of the animal. In fact, Kloek stated that a dog was capable of recognizing the odors of simple steroids, and that steroids belong to pheromonal substances. ${ }^{3)}$

Putative pheromones identified as steroid have been isolated from the male pig, ${ }^{10)}$ and $5 \alpha$-androsterone and testosterone could alter the action potential activity in the pig's olfactory bulb.5) Since the metabolites of steroids are excreted from the animal, the use of highly active simple steroids is not ideal. It is interesting, however, to consider the steroid effect of the olfactory receptors on the neuroendocrine structures. In fact, there is much evidence showing that olfaction affects mammalian behavior of reproduction. Accordingly, from data showing anatomical connections between the lateral hypothalamic area and the ORG,9) it can be said that the ORG neurons rcsponding to steroid odors would regulate the neuroendccrine system.

Acknowledgements. The authors wish to thank Prof. S. F. Takagi for encouraging this work, Dr. K. Imai for analysing radioactive stercids, Dr. K. Kishi for the histological check, and Mr. R. Cowan for revising the English expressions.

\section{References}

1) Eichenbaum, H. et al.: Brain Behav. Evol., 17, 255-275 (1980).

2) Hellon, R. F.: J. Physiol., 214, 12P (1971).

3) Kloek, J.: Psychiat. Neurol. Neurochir., 64, 309-344 (1961).

4) Kreiner, J.: J. Comp. Neurol., 116, 117-133 (1961).

5) MacLeod, N. et al.: Brain Res., 164, 323-327 (1979).

6) Onoda, N., and Iino, M.: Proc. Japan Acad., 56B, 300-305 (1980).

7) Onoda, N. et al.: ibid., 57B, 355-358 (1981).

8) Onoda, N., and Mori, K.: J. Neurophysiol., 44, 29-39 (1980).

9) Oomura, Y. et al.: Brain Res. Bull., 5, suppl. 4, 151-161 (1980).

10) Patterson, R. L. S.: Sci. Food Agric., 19, 31-88 (1968).

11) Sapolsky, R. M., and Eichenbaum, H.: Brain Behav. Evol., 17, 276-290 (1980).

12) Tanabe, T. et al.: J. Neurophysiol., 38, 1284-1296 (1975).

13) Yarita, H. et al.: ibid., 43, 69-85 (1980). 